\title{
ORIGINAL ARTICLE \\ Spinal Cord Essentials: the development of an individualized, handout-based patient and family education initiative for people with spinal cord injury
}

\author{
JJ Delparte ${ }^{1}$, BA Chau ${ }^{1}$, S Mills ${ }^{1}$ and AS Burns ${ }^{1,2}$
}

\begin{abstract}
Study design: Mixed (environmental scan, qualitative and semiquantitative).
Objectives: To develop a customizable patient and family education resource for people with spinal cord injury (SCl).

Setting: $\mathrm{SCl}$ rehabilitation center (Canada).

Methods: An environmental scan was conducted and stakeholder input sought to generate a list of potential handouts and inform content development. Staff members were recruited to draft handouts, which were later edited and finalized by the project team. Hardcopies of handouts were placed in cabinets throughout the rehabilitation center. Staff members were also trained/coached to facilitate and promote usage; which was then tracked for 2 years.

Results: One hundred and fifty-six potential handouts were identified; 83 deemed high priority. Seventy-two handouts addressing patient organization, self-management and community integration were finalized for phase 1 . At inpatient admission, patients receive a binder containing base materials. The binder is then customized and populated with pertinent handouts throughout the rehabilitation admission, and taken home at discharge for future reference. Handouts with the highest usage covered medical management (for example, bladder, bowel and pressure ulcers), mobility and activities of daily living. Handouts were also helpful for staff training and orientation, as well as increasing the confidence of staff providing education outside their immediate area of expertise.

Conclusion: Spinal Cord Essentials is a novel patient and family education initiative comprised of practical handouts that address many important aspects of SCl management. Handouts can be downloaded for free in four languages (English, French, Chinese and Farsi) at www.spinalcordessentials.ca. Additional handouts are planned for subsequent phases of the project.
\end{abstract}

Spinal Cord (2014) 52, 400-406; doi:10.1038/sc.2014.22; published online 18 March 2014

Keywords: spinal cord injuries; teaching materials; health education; health promotion

\section{INTRODUCTION}

Patient and family education is integral to spinal cord injury (SCI) rehabilitation. Information needs are particularly important for personal care, maximizing independence and preventing and managing secondary health complications; ${ }^{1-5}$ however, the psychological and physical effects of the injury can present challenges to learning readiness. ${ }^{5-7}$ Although the volume of information that patients are faced with during inpatient rehabilitation can be overwhelming, ${ }^{8}$ appropriate delivery of patient education is imperative to reduce and prevent secondary health complications. ${ }^{9-13}$

Due to different learning styles, educational materials should be available in multiple formats and forums to ensure access to information when the need arises. ${ }^{7}$ Wolfe et al. ${ }^{8}$ recommended that the optimal delivery of information during inpatient rehabilitation is through 'small chunks' of information, such as brief handouts. The purpose of this manuscript is to (1) describe development and implementation and (2) increase awareness of Spinal Cord Essentials, a patient and family education initiative at the University Health Network-Toronto Rehabilitation Institute (UHN-TRI, Toronto, ON, Canada).

\section{MATERIALS AND METHODS}

\section{Identification of content}

Current educational materials were identified at the UHN-TRI and other select rehabilitation centers in Canada and the United States. Identified resources included seven books: After and Beyond Spinal Cord Injury, ${ }^{14}$ Life After Spinal Cord Injury books, ${ }^{15,16}$ Craig Hospital SCI Handbook, ${ }^{17}$ Yes, You Can 4th Edition, ${ }^{18}$ Magee Rehabilitation SCI Patient-Family Teaching Manual, ${ }^{19}$ and Rehabilitation after a SCI: A Guide for You after SCI. ${ }^{20}$ In-house materials included: service-specific handouts, a document entitled Teaching Prior to Weekend Pass and a draft Discharge Handbook. An unstructured online search of SCI-related websites identified additional resources: RehabTeamSite, ${ }^{21}$ Spinal Cord Injury Information Pages, ${ }^{22}$ SCI rehabilitation checklists from various centers, the Consortium for Spinal Cord Medicine Consumer Guides, ${ }^{23}$ and SCI Rehabilitation Evidence (SCIRE) ${ }^{24}$ A Medline literature search (2000-2009) using the MeSH terms spinal cord injuries \& patient education yielded five articles addressing the educational needs of patients with SCI. ${ }^{1-5}$ The results of the above environmental scan were then used to generate a preliminary list of handouts for stakeholder feedback.

Two $1.5 \mathrm{~h}$ focus groups were conducted and led by a facilitator and a member of the project team: (1) a consumer focus group consisting of persons with SCI, an attendant care coordinator and family members $(n=7)$; and (2) a

${ }^{1} 1$ Lyndhurst Centre, Toronto Rehabilitation Institute, University Health Network, Toronto, Ontario, Canada and ${ }^{2}$ Department of Medicine, University of Toronto, Toronto, Ontario, Canada

Correspondence: JJ Delparte, 1Lyndhurst Centre, Toronto Rehabilitation Institute, University Health Network, 206-520 Sutherland Dr, Toronto, Ontario, Canada M4G 3V9. E-mail: jude.delparte@uhn.ca

Received 20 September 2013; revised 18 December 2013; accepted 4 February 2014; published online 18 March 2014 
multi-disciplinary staff focus group $(n=8)$. The groups addressed (1) usefulness of written materials, (2) potential additions and (3) format and methods of delivery. Participants signed informed consent and were compensated. Audio recordings were reviewed by the project team who extracted salient points. One-on-one meetings were conducted with professional practice leaders from each of the inpatient services: physiotherapy, occupational therapy, psychology, social work, therapeutic recreation, chaplaincy, dietary, nursing, respiratory therapy and community and homecare services. Representatives provided feedback about (1) handout additions, (2) high-priority handouts and (3) commonly encountered languages other than English.

\section{Development of educational materials}

Staff members were recruited to write one or more high-priority handouts. Prospective writers were provided guidelines, a template and instructions for creating easy-to-read handouts-limit handout length ( $<4$ pages), focus on practical information and minimize technical terms. As an incentive, staff members received gift certificates following the submission of initial drafts. Draft handouts were revised and returned to the original authors for final approval. An illustrator prepared required images, a photographer conducted photo shoots and a graphic designer participated in branding.

\section{Implementation}

Preparations included the development of promotional materials, a centerwide information session, meetings with inpatient services, training sessions and center-wide emails. On June 20, 2011 binders with a few key handouts were distributed to all inpatients. Designated filing cabinets containing the full series of handouts were placed in convenient locations throughout the hospital. Locations included the inpatient units, the outpatient department, the therapeutic gym, the center's resource center and staff offices. Colour-coded posters above the cabinets provided a quick reference for locating handouts divided into the following categories: Self-Management (Activities of Daily Living, Bladder, Bowel, Medical, Mobility, Skin, Emotional Health), Community Living (Equipment, Transportation, Financial Programs, Recreation, Home Environment and Care) and Organization (Figure 1).

On the launch day, a promotional event was held in the lobby to raise awareness. Staff training and orientation continued for approximately 2 months following initial launch. Activities included presentations at staff meetings, the creation of reference documents for those unable to attend and small group sessions to answer questions and highlight handouts relevant to specific disciplines. A binder with the full series of handouts was also provided to each service for reference and browsing. Handouts were also distributed on USB devices and made available online for public access in September 2012 at www.spinalcordessentials.ca.

\section{Evaluation}

Following the launch, surveys were distributed to patients and staff. The survey was adapted from a Website Evaluation Questionnaire ${ }^{25}$ to address the usability of the physical binder and handouts: structure, layout, comprehensiveness, relevance, organization, comprehensibility and userfriendliness. Open-ended questions solicited general feedback from staff and patients, including pros and cons. Written surveys were completed by staff, whereas patients participated in telephone surveys following discharge. Responses to open-ended questions were summarized by extracting themes. Mann-Whitney $U$ tests determined whether there were significant differences between patient and staff responses $(P \leqslant 0.05)$. Participants provided written or verbal consent, and data collection was approved by the UHN-TRI's Research Ethics Board.

Utilization of available handouts was tracked for 2 years following implementation in order to determine the most frequently used handouts. Tracking was performed every 3-6 weeks by noting when refills were required for each cabinet. Individual folders were refilled with 20-25 handouts, when there were less than approximately eight handouts.

\section{RESULTS}

Summary of content and operations

A list of 109 potential handouts was compiled from the environmental scan. An additional 23 handouts were recommended by the focus groups covering topics such as orientation to the rehabilitation center and surrounding area, available services after discharge (for example, housing and attendant care) and follow-up schedules for medical appointments. Attendees suggested that a binder with accompanying handouts would be ideal; however, materials should be available electronically for individuals who have difficulty manipulating a binder (for example, persons with tetraplegia).

Following one-on-one meetings and additional discussion within the project team, the final list consisted of 156 handouts with 83 designated as high priority. For phase 1, 57 writers contributed to writing and finalizing 72 handouts (Figure 2). Seven were introductory materials or worksheets with limited content. The complete list of available handouts is provided in the Appendix. Remaining highpriority handouts, addressing predominantly sexuality and pain management, require additional editing and will be made available in the second phase of the project.

The binder is organized into eight colored tabs: Planning, Medical, Self-Care, Mobility, Community, Finances, Contacts and Other. Binders initially contain a small number of handouts relevant to all inpatients. During the course of inpatient rehabilitation, each patient's binder is customized by incorporating additional materials relevant to that specific patient. To help patients organize their binders, individual handouts incorporate a colour-coded icon that corresponds to the recommended tab for filing in the binder. The majority of handouts have been translated into French, Chinese and Farsi, and can be downloaded without cost at www.spinalcordessentials.ca. Additional languages are planned in the future.

Spinal Cord Essentials has also been integrated into staff orientation. New staff, students and volunteers have found the handouts to be informative for their own professional development as well as understanding specific rehabilitation teachings, which occur at the center. Relevant handouts are made available at biweekly patient and family education workshops. Volunteers assist in day-to-day activities including restocking cabinets, preparation/delivery of binders to units, printing handouts and orientation of patients to the binder.

\section{Utilization}

Three hundred and twenty-one folder refills were documented in the 2 years following launch. Inpatient units and the patient resource center showed the highest usage with an average of 3.33 folder refills per month. The gym and outpatient services were refilled approximately once per month, whereas physiotherapy and occupational therapy services required refills approximately every other month. Handouts that showed the highest usage addressed medical management, mobility and activities of daily living (Table 1).

\section{Patient and staff feedback}

Twenty staff and 27 patients completed questionnaires. Ratings were high for all questionnaire scales (Figure 3), and no significant differences were found between staff and patient responses. Staff members generally commented that Spinal Cord Essentials was convenient, aided with teaching patients, improved consistency and quality of staff training and assisted with teaching outside one's immediate area of expertise. Some indicated that additional handouts were needed to enable them to take full advantage of the project. A few staff members indicated that usage of the materials had not yet been incorporated into their routine and that their patients would not 


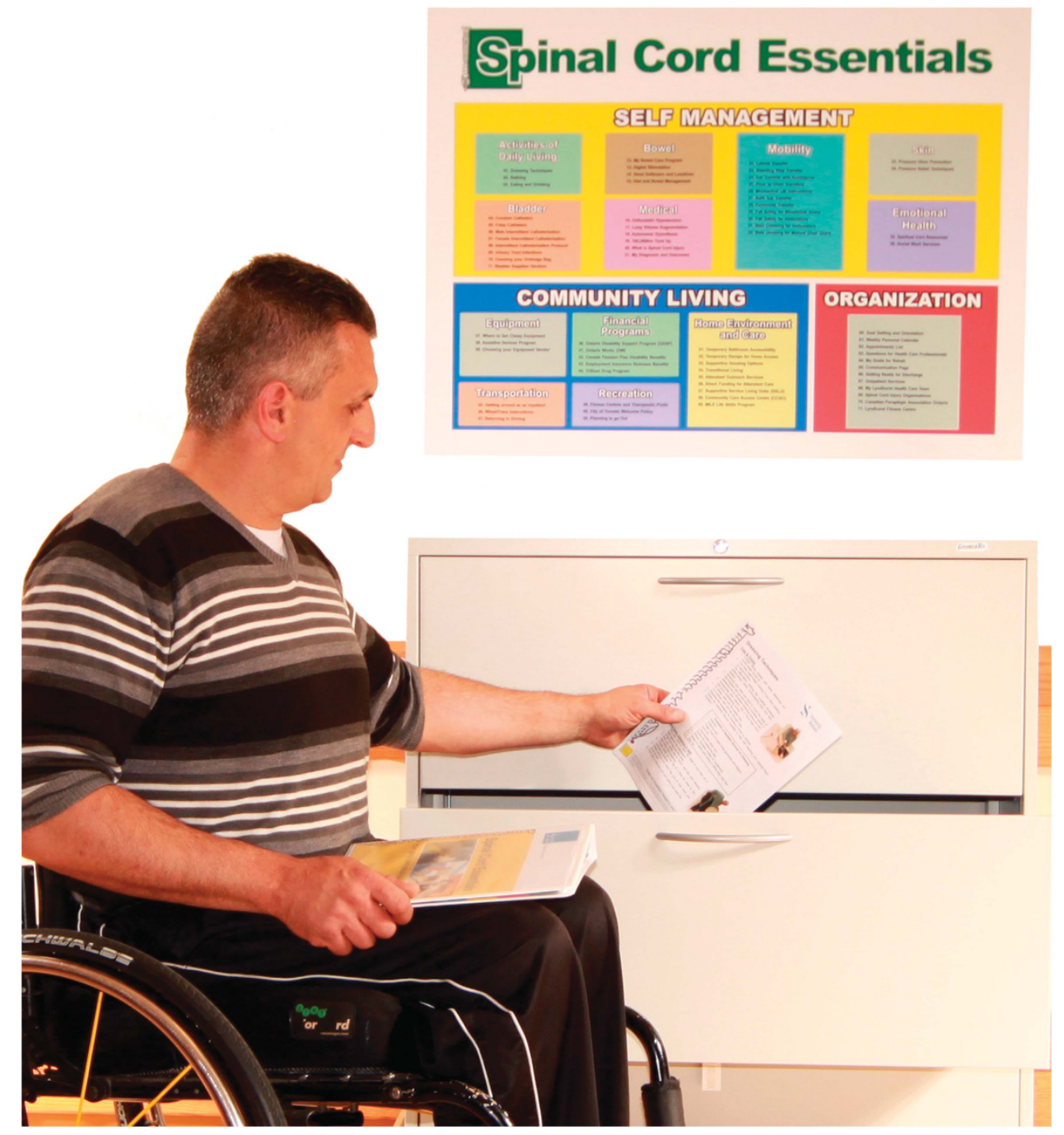

Figure 1 Poster and cabinet containing hard copies of the handouts used in multiple areas around the rehabilitation centre.

make good use of the materials. Patients found the binder helpful for organizing the various materials received. A few patients indicated that the binder was not helpful because of a preference to retrieve information online. Also, some patients indicated that staff should be more proactive in providing them with handouts during their stay.

\section{DISCUSSION}

Effective education provides individuals with SCI and their families with the knowledge and skills required to self-manage their condition and maintain health. Shorter rehabilitation lengths of stay ${ }^{26}$ reduce opportunities for teachable moments and increase the likelihood of patients being discharged before having acquired important knowledge and skills to prevent secondary health complications. ${ }^{5,6}$ It is therefore imperative that patient and family education be delivered efficiently and effectively. In recognition of the above, UHN-TRI augmented its efforts in this area by hiring a dedicated patient and family educator, opening a new SCI information center and expanding the patient and family education workshop series. Spinal Cord Essentials compliments each of these new efforts.
Ideally, patient education should be designed and delivered according to principles of adult learning theory, which help patients develop skills for self-directed lifelong learning. ${ }^{7}$ Redundancy and repetition spread across teaching strategies is optimal in order to accommodate different learning styles. ${ }^{5,6,27}$ Making materials available in multiple formats ensures that relevant information is accessible whether patients are at the rehabilitation center or in the community.

Spinal Cord Essentials is grounded in prior recommendations for optimizing patient education. Wolfe et al. ${ }^{8}$ recommended that an ideal educational resource would involve a customized patient binder, which would be populated as teachable moments arise. In addition, Spinal Cord Essentials adheres to the characteristics of effective patient education: ${ }^{28}$

- Primacy. Handouts are limited to important, practical information.

- Brevity. The handouts are brief (1-4 pages).

- Organization. Handouts contain clear headers and are organized into thematic categories; which guide filing and are displayed on informational posters.

- Specificity. Instructions are clearly outlined, bulleted and numbered when possible. 


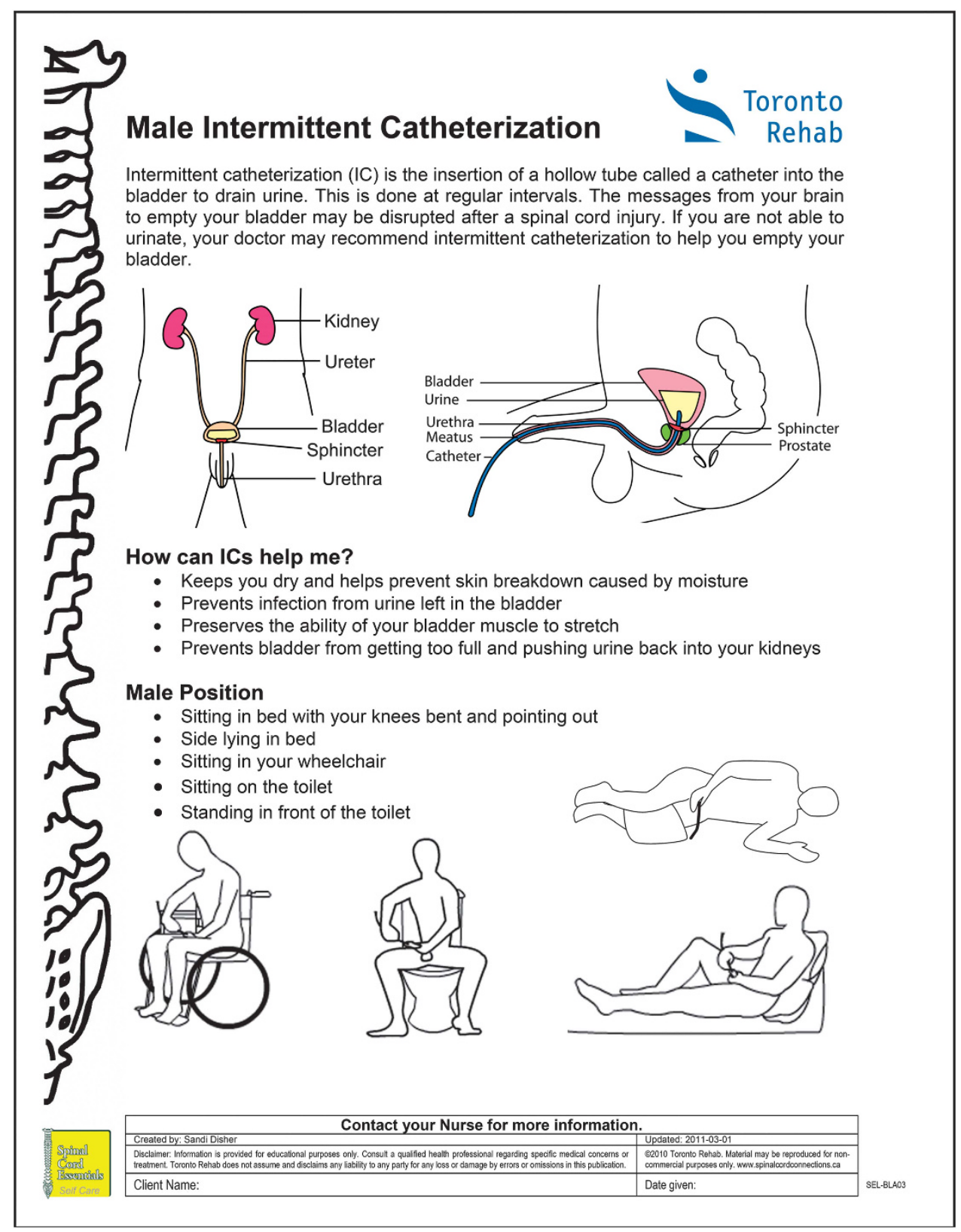

Figure 2 Example of handout layout.

Table 1 Handout usage across cabinet locations

\begin{tabular}{|c|c|c|}
\hline Patient units & $\begin{array}{l}\text { Resource center and } \\
\text { outpatients }\end{array}$ & OT/PT/GYM \\
\hline Diet and bowel management & IC protocol & Dressing techniques \\
\hline Male IC & Cleaning your drainage bag & Autonomic dysreflexia \\
\hline IC protocol & Autonomic dysreflexia & Standing step transfer \\
\hline Stool softeners and laxatives & Pressure ulcer prevention & Car transfer with assistance \\
\hline Community transportation & Outpatient services & Floor to chair transfers \\
\hline Urinary tract infections & Diet and bowel management & Fall safety for wheelchair users \\
\hline My bowel care program & Planning to go out & Pressure-relief techniques \\
\hline Autonomic dysreflexia & Digital stimulation & Where to get cheap equipment \\
\hline Pressure ulcer prevention & Pressure-relief techniques & Stairs for manual chair users \\
\hline Digital stimulation & - & Pressure ulcer prevention \\
\hline- & - & Lateral transfer \\
\hline
\end{tabular}

Abbreviation: IC, intermittent catheterization.

Handouts are listed in descending order of usage.
- Repetition. Materials are supplementary to workshops, point of care teaching and other available written materials.

- Literacy level. Handouts are written at an 8th grade reading level.

Spinal Cord Essentials is novel in that it differs from lengthier documents and books which are currently available. ${ }^{14-20}$ Lengthier documents are useful references but are not optimal for teaching specific skills in the moment. The format of Spinal Cord Essentials allows information to be delivered in a salient manner, while focusing on the practical. Matter et al. ${ }^{1}$ found that compared with books and public libraries, handouts and brochures are preferred formats for information delivery; however, the most commonly preferred method for information delivery was the internet. 


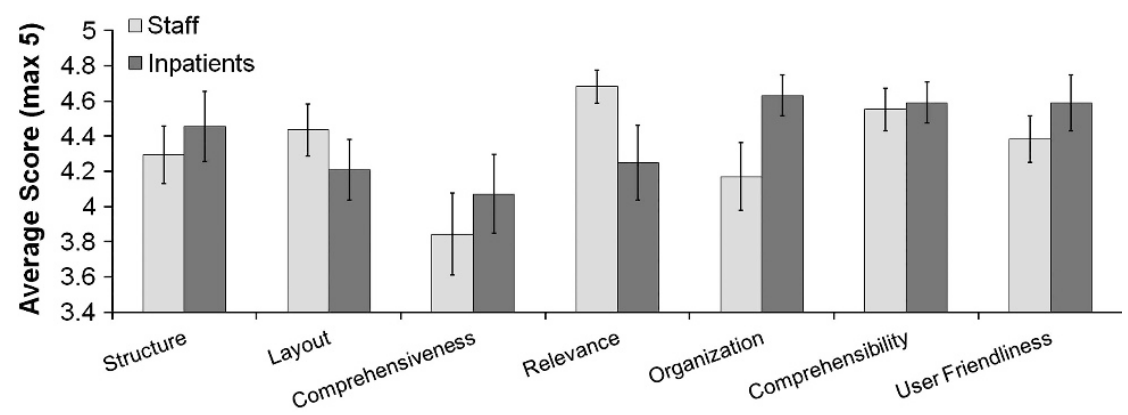

Figure 3 Average scores for patients and staff on the scales of the binder evaluation questionnaire. Error bars denote s.e.m.

Among community dwellers with SCI and other disabilities, there is an increased interest in internet-based health information resources. ${ }^{1,2,29-31}$ Although online learning can be helpful during inpatient rehabilitation, it is especially important following community integration. Patients may not be motivated or ready to learn about different aspects of their injury until a dire need arises.? The availability of a personalized reference binder (compiled during inpatient rehabilitation) and online materials help ensure information is accessible when needed. The handouts are also being used to train private caregivers in the specific care needs for people with SCI living in the community.

Traditionally, a limitation of internet-based resources has been the accuracy and quality of information. ${ }^{2,32}$ Increasingly, SCI health information resources published by reputable sources are becoming available online. ${ }^{18-23,33,34}$ Spinal Cord Essentials is available online as PDF files at www.spinalcordessentials.ca in English, French, Chinese and Farsi. An additional resource that has been integrated into the SCI rehabilitation program at UHN-TRI is www.SCI-U.ca, which consists of a series of 10 e-learning courses. ${ }^{33}$ The use of audio and visual media, expert opinions, peer interviews and endorsement by multiple SCI rehabilitation centers across Canada renders it a highly valued complementary educational resource for people with SCI.

The first phase of Spinal Cord Essentials addressed the most immediate aspects of inpatient rehabilitation including self-care, mobility, financial programs and home modifications. Handout usage has revealed great interest in medical management, mobility and activities of daily living. Prior studies have investigated the educational needs of persons with SCI, and personal care and potential health complications were often rated as most important. ${ }^{1-5}$ Additional areas of interest include aging, research, financial aid, support group information and community integration. ${ }^{3-5}$ Of the 156 candidate handouts that were identified, less than half have been brought to publication. Clear gaps in information still exist, notably in the areas of sexuality, medications and pain management. The next phase of Spinal Cord Essentials will prioritize these topics.

The initial emphasis was placed on assessing how the resource was received by staff and patients. Although the evaluation of the impact of Spinal Cord Essentials on the efficacy of patient and family education is being considered in the future, a significant barrier includes the relative lack of a validated outcome measure to assess patient learning about SCI. In addition, isolating the contribution of a single component of an institution-wide educational initiative may not be possible.

The high average scores on the usability questionnaire scales suggest that staff and patients were satisfied with most aspects of the resource. Scoring lowest was the question on comprehensiveness, which underscores the importance of releasing additional handouts in the future. This was reinforced through the qualitative feedback where some staff indicated that additional handouts would be required for them to take full advantage of Spinal Cord Essentials. Also of interest, some patients indicated that the binder did not align with their learning styles and that digital sources were preferred.

The physical presence of Spinal Cord Essentials (for example, cabinets and posters) has helped increase the general awareness of patient education. Furthermore, handouts are now frequently being used for staff training and orientation. Handouts helped increase the homogeneity of procedures taught across the center and have provided staff with the confidence to provide information to patients that is outside their immediate area of expertise; two common barriers to patient education. ${ }^{7,28}$

Written materials must be periodically revised and ongoing funding, infrastructure and staff are required. Spinal Cord Essentials has secured funding through sponsorship and vendor advertisements in the binders. At the time of this publication, no ads have been placed on the website; however, this is being considered. With the help of ongoing funding, the next phases of the project will be actualized including the release of new handouts, an update of the website and translation into additional languages.

\section{CONCLUSION}

Spinal Cord Essentials is a unique patient education resource comprised of a series of concise, practical handouts, which highlight specific ways in which individuals with SCI can self-manage their condition, troubleshoot financial and service needs and access community resources. It has been developed to complement existing strategies and resources and is freely available online at www.spinalcordessentials.ca.

\section{DATA ARCHIVING}

There were no data to deposit.

\section{CONFLICT OF INTEREST}

The authors declare no conflict of interest.

\section{ACKNOWLEDGEMENTS}

The creation of Spinal Cord Essentials was made possible through an Ontario Ministry of Health and Long Term Care Alternative Funding Plan Innovation Fund. 
1 Matter B, Feinberg M, Schomer K, Harniss M, Brown P, Johnson K. Information needs of people with spinal cord injuries. J Spinal Cord Med 2009; 32: 545-554.

2 Burkell JA, Wolfe DL, Potter PJ, Jutai JW. Information needs and information sources of individuals living with spinal cord injury. Health Info Libr J 2006; 23: 257-265.

3 Gontkovsky ST, Russum P, Stokic DS. Perceived information needs of communitydwelling persons with chronic spinal cord injury: findings of a survey and impact of race. Disabil Rehabil 2007; 29: 1305-1312.

4 May L, Day R, Warren S. Evaluation of patient education in spinal cord injury rehabilitation: knowledge, problem-solving and perceived importance. Disabil Rehabil 2006; 28: 405-413.

5 Manns PJ, May LA. Perceptions of issues associated with the maintenance and improvement of long-term health in people with SCI. Spinal Cord 2007; 45: 411-419.

6 Potter PJ, Wolfe DL, Burkell JA, Hayes KC. Challenges in educating individuals with $\mathrm{SCl}$ to reduce secondary conditions. Top Spinal Cord Inj Rehabil 2004; 10: 30-40.

7 May L, Day R, Warren S. Perceptions of patient education in spinal cord injury rehabilitation. Disabil Rehabil 2006; 28: 1041-1049.

8 Wolfe DL, Potter PJ, Swqueira KAJ. Overcoming challenges: the role of rehabilitation in education individuals with $\mathrm{SCl}$ to reduce secondary conditions. Top Spinal Cord Inj Rehabil 2004; 10: 41-50.

9 Hagglund KJ, Clark MJ, Schopp LH, Sherman AK, Acuff ME. Consumer-assistant education to reduce the occurrence of urinary tract infections among persons with spinal cord injury. Top Spinal Cord Inj Rehabil 2005; 10: 53-62.

10 Ljungberg I, Kroll T, Libin A, Gordon S. Using peer mentoring for people with spinal cord injury to enhance self-efficacy beliefs and prevent medical complications. J Clin Nurs 2011; 20: 351-358.

11 Rintala DH, Garber SL, Friedman JD, Holmes SA. Preventing recurrent pressure ulcers in veterans with spinal cord injury: impact of a structured education and follow-up intervention. Arch Phys Med Rehabil 2008; 89: 1429-1441.

12 Schubart J. An e-learning program to prevent pressure ulcers in adults with spinal cord injury: a pre- and post- pilot test among rehabilitation patients following discharge to home. Ostomy Wound Manage 2012; 58: 38-49.

13 Cardenas DD, Hoffman JM, Kelly E, Mayo ME. Impact of a urinary tract infection educational program in persons with spinal cord injury. J Spinal Cord Med 2004; 27: 47-54.

14 Canadian Spinal Research Organization. After and Beyond Spinal Cord Injury-A resource manual to help guide you from rehabilitation back into the community. Canadian Spinal Research Organization: Richmond Hill, ON, 2002.

15 Canadian Paraplegic Association. Life After Spinal Cord Injury_Incomplete spinal cord injury. Canadian Paraplegic Association: Winnipeg, MB, 2001.

16 DeCorwin S, Filiatrault L, Maisonneuve C, Renald M, Savoie D, Staltari C et al. Life after spinal cord injury. Saint-Leonard, QC Association des paraplégiques du Québec 2002.

17 Craig Hospital. Craig Hospital Spinal Cord Injury Handbook. Craig Hospital: Englewood, CO, 2003
18 Burns SP, Hammond MC. Yes, you Can! A Guide to Self-Care for Persons with Spinal Cord Injury, 4th ed. Paralyzed Veterans of America: Washington, DC, 2009.

19 Regional Spinal Cord Injury Center of the Delaware Valley. Magee Rehabilitation Spinal Cord Injury Patient-Family Teaching Manual. Thomas Jefferson University: Philadelphia, PA, 2009.

20 Hamilton Health Sciences. Rehabilitation after a spinal cord injury: a guide for you after spinal cord injury. Hamilton Health Sciences: Hamilton, ON, 2011.

21 Burrows SC, Moore KM, Lemkau HL Jr. Creating a web-accessible, point-of-care, teambased information system (PointTIS): the librarian as publisher. Bulletin of the Medical Library Association 2001; 89: 154-164.

22 Spinal Cord Injury Information Pages (cited 2013 June 28). Available from: www.sci-info-pages.com.

23 Consortium for Spinal Cord Medicine. Consumer Guides (Aug 29, 2013). Available from: www.scicpg.org.

24 Eng J, Teasell R, Miller W, Wolfe DL, Townson AF, Aubut JA et al. Spinal cord injury rehabilitation evidence: methods of the SCIRE systematic review. Top Spinal Cord Inj Rehabil 2007; 13: 1-10.

25 Elling S, Lentz L, De Jong M. Website Evaluation Questionnaire: development of a research-based tool for evaluating informational websites. Lecture Notes in Computer Science 2007; 4656: 293-304.

26 Burns AS, Yee J, Flett HM, Guy K, Cournoyea N. Impact of benchmarking and clinical decision making tools on rehabilitation length of stay following spinal cord injury. Spinal Cord 2013; 51: 165-169.

27 Chase TM. Learning styles and teaching strategies: enhancing the patient education experience. SCl Nurs 2001; 18: 138-141.

28 Lanig IS. Principles of effective patient education: General considerations. In: Lanig IS (ed.) A practical guide to health promotion after spinal cord injury. Aspen Publishers: Gaithersburg, MD, 1996, pp 34-39.

29 Hauber RP, Vesmarovich S, Dufour L. The use of computers and the Internet as a source of health information for people with disabilities. Rehabil Nurs 2002; 27: 142-145.

30 Edwards L, Krassioukov A, Fehlings MG. Importance of access to research information among individuals with spinal cord injury: results of an evidenced-based questionnaire. Spinal Cord 2002; 40: 529-535.

31 Goodman N, Jette AM, Houlihan B, Williams S. Computer and internet use by persons after traumatic spinal cord injury. Arch Phys Med Rehabil 2008; 89: 1492-1498.

32 Risk A, Petersen C. Health information on the internet: quality issues and international initiatives. Jama 2002; 287: 2713-2715.

33 Shepherd JD, Badger-Brown KM, Legassic MS, Walia S, Wolfe DL. SCI-U: e-learning for patient education in spinal cord injury rehabilitation. J Spinal Cord Med 2012; 35: 319-329.

34 Hoffman J, Salzman C, Garbaccio C, Burns SP, Crane D, Bombardier C. Use of on-demand video to provide patient education on spinal cord injury. J Spinal Cord Med 2011; 34: 404-409. 


\section{APPENDIX}

\section{Self Management}

\section{Activities of Daily Living}

Dressing Techniques

Bathing

Eating and Drinking

\section{Bladder}

Condom Catheters

Foley Catheters

Male Intermittent Catheterization

Female Intermittent Catheterization

Intermittent Catheterization Protocol

Urinary Tract Infections

Cleaing Your Drainage Bag

Bladder Supply Vendors

\section{Bowel}

My Bowel Care Program

Digital Stimulation

Stool Softeners and Laxatives

Diet and Bowel Management

\section{Emotional Health}

Spiritual Care Resources

Social Work Services

\section{Medical}

Orthostatic Hypotension

Lung Volume Augmentation

Autonomic Disreflexia

100,000 km Tune Up

What is Spinal Cord Injury

My Diagnosis and Outcomes

\section{Skin}

Pressure Ulcer Prevention

Pressure Relief Techniques

\section{Mobility}

Lateral Transfer

Standing Step Transfer

Car Transfer with Assistance

Floor to Chair Transfer

Mechanical Lift Instructions

Bath Tub Transfer

Commode Transfer

Fall Safety for Wheelchair Users

Fall Safety for Amubulators

Stair Climbing for Ambulators

Stair Climbing for Manual Chair Users

\section{Community Living}

\section{Equipment}

Where to Get Cheap Equipment

Assistive Devices Program

Choosing Your Equipment Vendor

\section{Transportation}

Getting Around as an Inpatient

WheelTrans Instructions

Returning to Driving

\section{Recreation}

Fitness Centres and Therapeutic Pools

City of Toronto Welcome Policy

Planning To Go Out

\section{Home Environment and Care}

Temporary Bathroom Accessibility

Temporary Ramps for Home Access

Supportive Housing Options

Transitional Living

Attendant Outreach Services

Direct Funding for Attendant Care

Supportive Service Living Units

Community Care Access Centre

MILE Life Skills Program

\section{Financial Programs}

Ontario Disability Support Program

Ontario Works

Canada Pension Plan Disability Benefits

Employment Insurance Sickness Benefits

Trillium Drug Program

\section{Organization}

Introduction to Spinal Cord Essentials

Acknowledgements

Weekly Personal Calendar

Appointments List

Questions for Health Care Professionals

My Goals for Rehab

Communication

Getting Ready for Discharge

Outpatient Services

My Spinal Cord Rehab Health Team

Contacts

Canadian Paraplegic Association Ontario

Lyndhurst Centre Fitness Centre 